\title{
Long-term results of intertrochanteric varus osteotomy for dysplastic osteoarthritis of the hip
}

\author{
Jochen Zweifel • Wolfgang Hönle • Alexander Schuh
}

Received: 17 July 2009 /Revised: 22 August 2009 /Accepted: 23 August 2009 /Published online: 12 September 2009

(C) Springer-Verlag 2009

\begin{abstract}
The beneficial role of intertrochanteric varus or valgus osteotomy in the treatment of hip osteoarthrosis has been recognised since the 1920s. Even today, it has an important role to delay the need for hip replacement in younger patients. The purpose of this study was to evaluate the long-term results of intertrochanteric varus osteotomy for dysplastic osteoarthritis of the hip. Between 1980 and 1995, 52 hips with dysplastic osteoarthritis underwent an intertrochanteric varus osteotomy. All patients could be followed up after 17.8 years. Radiographic assessment included centre-edge (CE) angle of Wiberg and collodiaphyseal (CCD) angle. Dysplastic hip dislocation was classified according to Crowe. Osteoarthritis was graded according to Tönnis. Preoperative osteoarthritis was Tonnis grade 1 in 19 hips, grade 2 in 28 and grade 3 in five. Complications were also documented. Clinical evaluation included score according to Merle d'Aubigné, Harris Hip Score and range of motion. Six of 52 patients underwent total hip replacement (THR) within five years after
\end{abstract}

\section{J. Zweifel}

Research Unit, Neumarkt Hospital,

92318 Neumarkt, Germany

W. Hönle

Department of Orthopedic Surgery, Neumarkt Hospital, 92318 Neumarkt, Germany

\author{
A. Schuh \\ Research Unit Orthopaedics and Surgery, Neumarkt Hospital, \\ 92318 Neumarkt, Germany

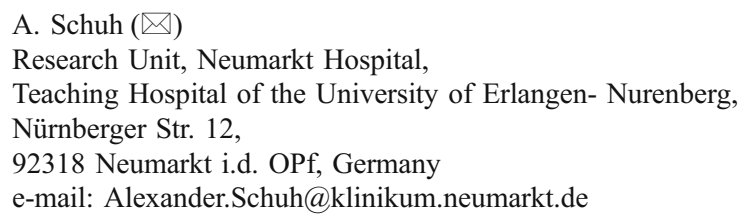

intertrochanteric osteotomy. In these cases, preoperative osteoarthritis was grade 3 in four cases and grade 2 in one case according to Tönnis. Twenty-five of 52 patients underwent THR after an interval of five to ten years after intertrochanteric osteotomy. In these cases, preoperative osteoarthritis was grade 3 in one case and grade 2 in 24 cases according to Tönnis. Sixteen of 52 patients underwent THR after an interval of ten to 15 years after intertrochanteric osteotomy. In these cases, preoperative osteoarthritis was grade 2 in four cases and grade 1 in 12 cases according to Tönnis. In five patients the hip was functioning well after a period longer than 15 years. In all these cases osteoarthritis was grade 1 according to Tönnis. In $40.4 \%$ of patients, THR was delayed longer than ten years. The osteotomy performed well at a mean time of 9.7 years after the procedure (range 3-21). Intertrochanteric varus osteotomy for dysplastic osteoarthritis of the hip should be considered in early or mild osteoarthritic changes of the hip. Good prognosis with delay of THR of more than ten years can be expected to exceed $40 \%$, even when indications are less than optimum.

\section{Introduction}

Total hip replacement (THR) is a very successful treatment option for osteoarthritis of the hip in the elderly, but it is uncertain whether this also holds true for younger patients. In the short term, THR shows excellent results; but in the long term, high revision rates have been reported $[5,10]$. The beneficial role of intertrochanteric varus or valgus osteotomy in the treatment of hip osteoarthrosis has been recognised since the 1920s [10]. Even today, it is important to delay the need for hip replacement in younger patients [5]. The rationale of hip osteotomies, pelvic or femoral, is to 
improve the mechanical environment and the joint congruency before the patient is highly symptomatic. Theoretically, most of the beneficial effects of osteotomies are believed to be the result of reduced load and stress on the hip [10, 13, 14]. Additionally, it has been postulated that the damaged cartilage is reparable if the load and stress can be reduced. There have been few reports regarding long-term results of intertrochanteric varus osteotomy for treatment of dysplastic osteoarthritis of the hip [7, 8]. The purpose of this study was to evaluate the long-term results of intertrochanteric varus osteotomy for dysplastic osteoarthritis of the hip.

\section{Material and method}

Between 1980 and 1995, 52 hips with dysplastic osteoarthritis underwent an intertrochanteric varus osteotomy. Average patient age was 36.4 (range 20-44) years. There were 48 women and four men. Average body mass index (BMI) preoperatively was 23.5 (range 17. 6-33.6).

\section{Operation technique}

In the preoperative planning, all patients had functional radiographs in adduction and abduction. The decision to perform an osteotomy, including which type of osteotomy, was based on radiographic improvement of congruency and containment. All osteotomies were performed using a standard lateral approach [10]. The capsule was opened, and guide wires were inserted into the inferior half of the femoral head, followed by insertion of the chisel through the distal part of the trochanteric bed. The guide was set at the necessary correction angle according to preoperative plan. Femoral osteotomy was performed without removing a wedge of bone. Fixation was performed with an $\mathrm{AO} 90^{\circ}$ blade-plate. The plate was removed one year after the index operation. In all cases, a varus osteotomy was performed. In 16 cases, an additional extension and derotation was necessary. In one case, an additional periacetabular osteotomy according to Wagner was performed at plate removal. One acetabular bone cyst had to be resected, including synovectomy of the affected hip joint, at plate removal. In seven cases, a shelf acetabuloplasty and in two cases a displacement of the greater trochanter was also performed.

\section{Radiological assessment}

The radiographic series of the operated hip for each patient was analysed. The assessments were made at the initial presentation, at 1 year and at latest follow-up. Osteoarthritis was graded according to Tönnis [18]. The centre-edge (CE) angle of Wiberg [20] and collodiaphyseal (CCD) angle were measured. Dysplastic hip dislocation was classified according to Crowe [1]. Preoperative osteoarthritis was Tonnis grade 1 in 19 hips, grade 2 in 28 and grade 3 in 5. Complications were also documented. Clinical evaluation included score according to Merle d'Aubigné [12], Harris Hip Score [3] and range of motion.

\section{Results}

All patients could be followed up after 17.8 years. Six of 52 patients underwent THR within five years after intertrochanteric osteotomy. In these cases, preoperative osteoarthritis was grade 3 in four cases and grade 2 in one case according to Tönnis. Twenty-five of 52 patients underwent THR after an interval of five to ten years after intertrochanteric osteotomy. In these cases, preoperative osteoarthritis was grade 3 in one case and grade 2 in 24 cases according to Tönnis. Sixteen of 52 patients underwent THR after an interval of ten to 15 years after intertrochanteric osteotomy. In these cases, preoperative osteoarthritis was grade 2 in four cases and grade 1 in 12 cases according to Tönnis. In five patients, the hip was functioning well after more than 15 years. In all these cases, preoperative osteoarthritis was grade 1 according to Tönnis. In $40.4 \%$ of cases, THR was delayed more than ten years. The osteotomy performed well at a mean of 9.7 (range three to 21) years after the procedure. Score according to Merle d'Aubigné was 12.6 preoperatively, 14.3 at the one year follow-up, 13.4 at the 5-year follow-up, 13.0 at the 10-year follow-up and 12.9 at the latest follow-up after 17.8 years. Harris Hip Score was comparable, with a mean of 64.3 preoperatively, 77.0 at the one year follow-up, 72.4 at the five year follow- up, 69.4 at the ten year follow- up and 69.2 at the latest follow-up after 17.8 years. Postoperative complications included one superficial and one deep wound infection requiring revision, one nonlethal pulmonary embolism, one delayed bone healing, one pseudarthrosis requiring revision surgery and

Table 1 Mean range of motion (FU: follow-up)

\begin{tabular}{|c|c|c|c|c|c|}
\hline & Before operation & 1-year FU & 5-year FU & 10-year FU & Latest FU \\
\hline Extension/flexion & $0 / 8 / 104^{\circ}$ & $0 / 4 / 102^{\circ}$ & $0 / 6 / 95^{\circ}$ & $0 / 8 / 98^{\circ}$ & $0 / 10 / 96^{\circ}$ \\
\hline Abduction/adduction & $27 / 0 / 22^{\circ}$ & $33 / 0 / 23^{\circ}$ & $26 / 0 / 20^{\circ}$ & $24 / 0 / 19^{\circ}$ & $23 / 0 / 17^{\circ}$ \\
\hline External rotation/internal rotation & $23 / 0 / 18^{\circ}$ & $28 / 0 / 13^{\circ}$ & $23 / 0 / 12^{\circ}$ & $24 / 0 / 11^{\circ}$ & $22 / 0 / 10^{\circ}$ \\
\hline
\end{tabular}


one heterotopic ossification (HTO) with limited range of motion requiring removal of HTO. Range of motion preoperatively and at each follow-up are presented in Table 1.

\section{Radiological assessment}

Preoperatively, mean CCD angle measured $146^{\circ}$ and mean $\mathrm{CE}$ angle measured $18^{\circ}$. Postoperatively, mean CCD angle measured $125^{\circ}$ and mean CE angle measured $22^{\circ}$. According to the classification of Crowe [1], 51 hips were $\mathrm{I}^{\circ}$ and one hip $\mathrm{II}^{\circ}$.

\section{Discussion}

Harris reported that once pain and roentgenographic degeneration start in dysplastic hips, they progress rapidly [2]. Femoral osteotomy for osteoarthritis of the hip has been carried out since the 1920-1930s [10]. A recent survey by Haverkamp et al. [4] showed that, even among experts, the use of intertrochanteric osteotomies is declining. The declining role of osteotomies could also be caused by patient preferences. Modern patients mostly desire a quick result, which is easier to obtain with a THR than with an osteotomy. A second patient preference could be that they are no longer satisfied with the good results that can be obtained with an osteotomy but want the excellent results that a THR could probably provide. It is important for the orthopaedic surgeon to inform patients of the long-term effects that this decision could have and to advise osteotomies for patients who could benefit from them [4]. In the short and midterm, THR shows excellent results; but in the long term, high revision rates have been reported. In young patients with high physical demands, jointpreserving procedures are more valuable today than ever, not only as a means to prevent osteoarthrosis but also to treat it by reducing the mechanical overload. Realigning varus or valgus osteotomies of the proximal femur are the most well-established joint-preserving procedures applied [4-11, 14, 15, 19]. The rationale for these procedures is the reduction of stress throughout the cartilage surfaces in a hip that is compromised mechanically [10]. The aim of intertrochanteric osteotomies is to delay THR. THR following proximal femoral osteotomies is possible with good to excellent results. Recently, Suzuki et al. published excellent clinical and radiological mid-term results for cementless THR after previous intertrochanteric valgus osteotomy [17]. As the configuration of the proximal femur may be changed, modular stems may be suitable to deal with the residual deformity and sclerosis in the metaphysial region after previous intertrochanteric valgus osteotomy
[17]. Another treatment option in these cases is the use of a cone prosthesis [16].

In our series, THR was delayed more than ten years in $40.4 \%$ of patients. Osteotomy performed well at a mean time of 9.7 (range 3-21) years after the procedure. Ito et al. [7] recently published long-term results for intertrochanteric varus osteotomy for dysplastic osteoarthritis of the hip. The cumulative rates of survival were $81 \%$ at ten years, $60 \%$ at 20 years and $50 \%$ at 25 years. Excellent or good clinical results were obtained in 23 hips (42\%). Compared with these results, our survival rate was much worse. We emphasise, however, that in our series, there were more cases of osteoarthritis grades 2 and 3 according to Tönnis. We agree with Ito et al., who stated that intertrochanteric osteotomy is worthwhile in cases with grades 0,1 or 2 osteoarthritis with a good spherical femoral head and mild dysplasia. Osteotomy might not be indicated for patients with grade 3 osteoarthritis or for those with severe acetabular dysplasia. Iwase et al. postulated that acetabular coverage influences the final outcome of intertrochanteric osteotomy [8]. Watillon et al. [19] concluded that osteotomies can provide excellent and lasting results when the indication is correct and the surgical procedure is carried out properly. They recommended a varus osteotomy for coxa valga with subluxation presenting with a dense triangle at the edge of the socket and congruent joint surfaces in abduction, and a valgus osteotomy when the femoral head and the acetabulum are considerably deformed with a dense triangle at the edge of the socket and with the joint surfaces congruent in adduction only.

\section{Conclusion}

We conclude that intertrochanteric varus osteotomy for dysplastic osteoarthritis of the hip should be considered in early or mild osteoarthritic changes of the hip. Good prognosis with delay of THR of more than ten years can be expected to exceed $40 \%$, even when indications are less than optimal.

\section{References}

1. Crowe JF, Mani VJ, Ranawat CS (1979) Total hip replacement in congenital dislocation and dysplasia of the hip. J Bone Joint Surg 61-A:15-23

2. Harris WH (1986) Etiology of osteoarthritis of the hip. Clin Orthop Relat Res 213:20-33

3. Harris WH (1969) Traumatic arthritis of the hip after dislocation and acetabular fractures: treatment by mold arthroplasty. An endresult study using a new method of result evaluation. J Bone Joint Surg Am 51-A:737-755

4. Haverkamp D, Eijer H, Besselaar PP, Marti RK (2008) Awareness and use of intertrochanteric osteotomies in current clinical practice. An international survey. Int Orthop 32:19-25 
5. Haverkamp D, Eijer H, Patt TW, Marti RK (2006) Multi directional intertrochanteric osteotomy for primary and secondary osteoarthritis-results after 15 to 29 years. Int Orthop 30:15-20

6. Haverkamp D, Marti RK (2005) Intertrochanteric osteotomy combined with acetabular shelfplasty in young patients with severe deformity of the femoral head and secondary osteoarthritis. A long-term follow-up study. J Bone Joint Surg Br 87-Br:25-31

7. Ito H, Matsuno T, Minami A (2005) Intertrochanteric varus osteotomy for osteoarthritis in patients with hip dysplasia: 6 to 28 years followup. Clin Orthop Relat Res 433:124-128

8. Iwase T, Hasegawa Y, Kataoka Y, Matsuda T, Iwata H (1995) Long-term results of intertrochanteric varus osteotomy for arthrosis of the dysplastic hip (over 10 years' follow-up). Arch Orthop Trauma Surg 114:243-247

9. Kawate K, Tanaka Y, Ohmura T, Hiyoshi N, Yajima H, Tomita Y, Takakura Y (2004) Twenty-five years followup of patients who had valgus osteotomy for arthritic hips. Clin Orthop Relat Res 426:151-158

10. Koulouvaris P, Stafylas K, Aznaoutoglou C, Zacharis K, Xenakis $T$ (2007) Isolated varus intertrochanteric osteotomy for hip dysplasia in 52 patients: long-term results. Int Orthop 31:193-198

11. McGrory BJ, Estok DM 2nd, Harris WH (1998) Follow-up of intertrochanteric osteotomy of the hip during a 25-year period. Orthopedics 21:651-653
12. Merle d'Aubigné R, Postel M (1954) Functional results of arthroplasty with acrylic prosthesis. J Bone Joint Surg Am 36:451-475

13. Millis MB, Kim YJ (2002) Rationale of osteotomy and related procedures for hip preservation: a review. Clin Orthop Relat Res $108-121$

14. Nissen KI (1963) The arrest of early primary osteoarthritis of the hip by osteotomy. Proc R Soc Med 56:1051-1060

15. Santore RF, Turgeon TR, Phillips WF 3rd, Kantor SR (2006) Pelvic and femoral osteotomy in the treatment of hip disease in the young adult. Instr Course Lect 55:131-144

16. Schuh A, Schraml A, Hohenberger G (2009) Long-term results of the Wagner cone prosthesis. Int Orthop 33:53-58

17. Suzuki K, Kawachi S, Matsubara M, Morita S, Jinno T, Shinomiya K (2007) Cementless total hip replacement after previous intertrochanteric valgus osteotomy for advanced osteoarthritis. J Bone Joint Surg Br 89:1155-1157

18. Tönnis D (1982) Congenital hip dislocation. Thieme-Stratton Inc, New York

19. Watillon M, Maquet P (1990) Indications for femoral intertrochanteric osteotomy for arthritis sequelae of hip dysplasia. Acta Orthop Belg 56:371-377

20. Wiberg G (1939) Studies on dysplastic acetabula and congenital subluxation of the hip joint. Acta Orthop Scand (Suppl) 58:29-37 